\title{
ASPLENIUM BALEARICUM SHIVAS EN LA ISLA DE MENORCA
}

\author{
J.A. ROSELLO \& M. SERRA
}

RESUMEN: Se estudia la variabilidad morfológica de Asplenium balearicum Shivas en la isla de Menorca, comparándose con los datos conocidos de las poblaciones italianas. Se esboza la ecología de la especie, con especial atención a sus preferencias edafológicas.

SUMMARY: The morfological variation of Asplenium balearicum Shivas from Menorca is studied and some observations about its ecology are given.

Recientemente dimos a conocer el redescubrimiento de Asplenium balearicum Shivas en las Islas Baleares (Rosselló et a1., 1986) cuya localización insular era desconocida. En dicho trabajo cartografiamos su distribución balear conocida y señalamos, muy sucintamente, algunos datos ecológicos. El estudio de abundante material de herbario, asi como numerosas observaciones realizadas en el campo nos llevó a considerar que la variabilidad fenotipica de A. balearicum era cuando menos notable. Presentamos en esta contribución el estudio morfológico de la especie y aportamos datos sobre su ecologia, desconocidos incluso en las poblaciones italianas señaladas por Nardi (1983).

\section{RESULTADOS}

En Menorca, A. balearicum presenta una notable variabilidad, hecho que sin duda ha dado lugar a confusiones con otras especies del complejo A. obovatum Viv. La longitud de la fronde, el número de pínnulas y la forma de las pinnas y pinnulas han resultado ser los caracteres más variables (fig. 1). Un gran número de formas intermedias enlazan los extremos de dicha variación. Hemos podido observar sobre el terreno que los ejemplares más pequeños, con frondes estrechamente triangulares, escaso número de pínnulas y con aspecto similar a frondes depauperadas de A. obovatum, son aquellos que generalmente se encuentran en las grietas de las rocas próximas al mar, en sitios expuestos. Mientras que en los lugares más resguardados y de hábito terrícola los ejemplares predominantes son mayores, aproximándose morfológicamente a A. onopteris L.

Los trasplantes de ejemplares de A. balearicum de Favaritx y Sa Bassa Verda no sobrevivieron el tiempo suficiente para observar qué caracteres morfológicos eran susceptibles de 
modificación en cultivo.

No nos ha sido posible comparar ejemplares de procedencia italiana con los de Baleares, aunque por la descripción dada por Nardi (1983) se insinúan algunas diferencias significativas. En la tabla 1 se comparan los caracteres más distintivos de A. balearicum así como sus márgenes de variación según la descripción original de Shivas (1969), Nardi (1983), Nogueira \& Ormonde (1986) y nuestras observaciones.

Destaca, en general, que las dimensiones de los macrocaracteres de los ejemplares de Menorca son sensiblemente inferiores a los de los ejemplares italianos. Las medidas de la longitud de la fronde, longitud del peciólo y dimensiones de las pinnas mayores se sitúan en los limites inferiores de variación observados por Nardi. Los ejemplares baleáricos presentan, principalmente, la lámina dos veces dividida (muy raramente 3 pinnada) al contrario que las poblaciones italianas donde predominan los ejemplares con segmentos de tercer orden.

El color del raquis de la cara abaxial varia en el seno de las poblaciones menorquinas desde enteramente verde a parcialmente castaño, en este caso la longitud pigmentada del raquis no sobrepasa $1 / 3$ de la longitud del mismo. Las diferencias de este carácter respecto a las poblaciones italianas y a la diagnosis original puede deberse al reducido número de ejemplares examinados por dichos autores, aunque no creemos que la coloración del raquis sea un carácter determinante en la discriminación de táxones en el complejo A. adiantum-nigrum $\mathrm{L}$.

En todas las poblaciones de A. balearicum examinadas 1a pínnula basal proximal acroscópica es mayor que las restantes mientras que en los ejemplares italianos es la pínnula basioscópica la que es de mayor tamaño. Este carácter, cuyo definitivo valor taxonómico nos es difícil valorar por el momento, no merecería mayor comentario si no fuera por el hecho de que es un carácter distintivo utilizado en la diagnosis de A. x bouharmontii Badré \& Prelli (A. obovatum x A. onopteris), Badré et al. (1981), predecesor híbrido de A. balearicum, y ha sido utilizado para separar dicho taxon de A. balearicum. En los ejemplares de A. x bouharmontii de Port Cros y Pantelleria y en los de A. balearicum de Menorca la pinnula proximal basal acroscópica es mayor que las otras, en tanto que en las islas italianas es la basioscópica. De este modo la distinción entre A. $x$ bouharmontii y A. balearicum, en ausencia de datos cariológicos, debería basarse exclusivamente en las modificaciones morfológicas de las estructuras reproductoras: esporangios y esporas.

Las diferencias entre las dimensiones esporales proporcionadas por Shivas (1969) y Nardi (1983) y nuestros datos creemos que deben ser atribuidas a cuestiones de método. Nuestras mediciones se obtuvieron sobre 30 esporas por ejemplar ( 9 ejemplares provenientes de 5 poblaciones), no acetolizadas, montadas en glicerogelatina y medidas al cabo de 20 días, excluyendo el perisporio. Shivas (1969) no precisa ni el medio de montaje ni la delimitación del parámetro a medir. Nardi (1983) monta las esporas en una solución acuosa de glicerina al $40 \%$, sin especificar tampoco si incluye el perisporio en las mediciones. Si nuestras observaciones del tamaño esporal se modifican con un factor de corrección ( +10 ; similar a la parte de perisporio no tenida en cuenta) los intervalos de variación son más o menos coincidentes y las medias idénticas, ajustándose a los tamaños obtenidos por Nogueira \& Ormonde 
Asplenium balearicum en Menorca
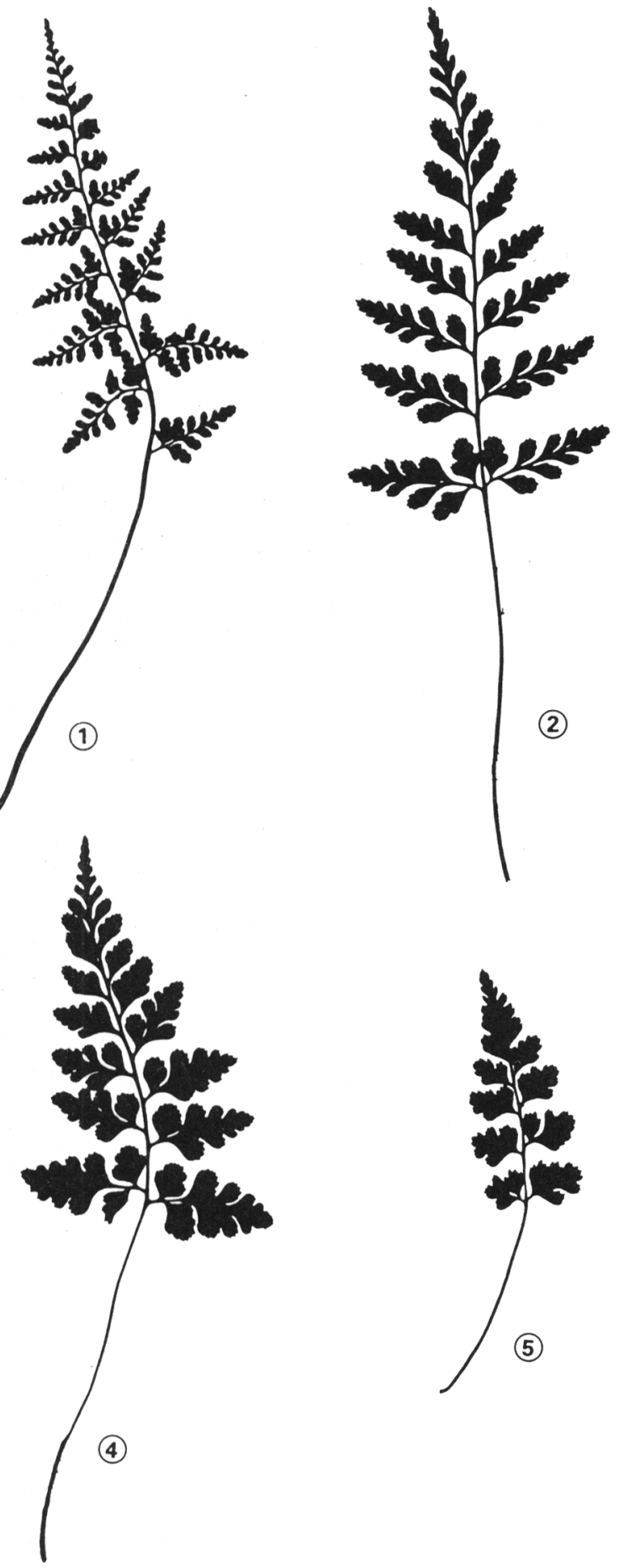

Figura 1.- Siluetas (fotocopias) de frondes fértiles de A. balearicum de diversas localidades de Menorca, 1 - Sa Bassa Verda, 2 - Montgofre Nou, 3-7 Favaritx.
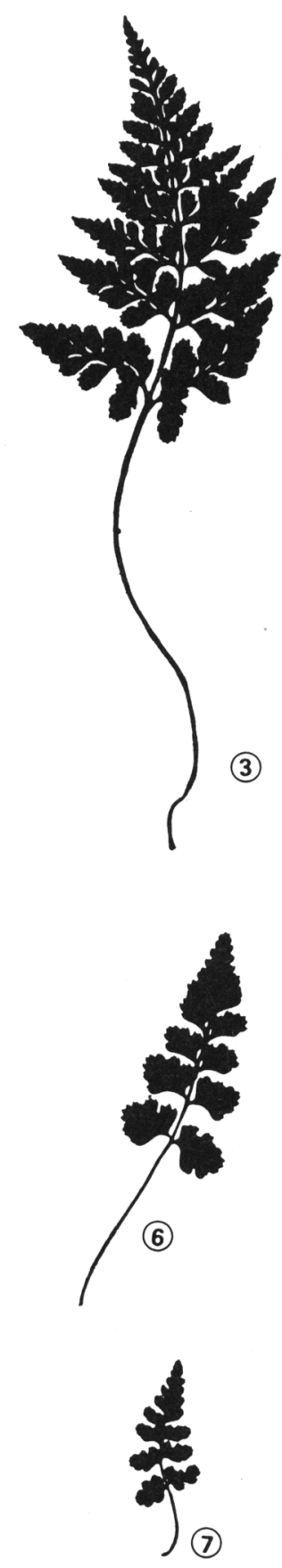
(1986) y por Ferrarini et al., (1986). Las escasas medidas efectuadas por nosotros sobre las esporas de A. onopteris coinciden con los limites proporcionados por Roberts (1979) y apuntan a que no es posible una clara separación entre A. balearicum y A. onopteris en base al tamaño esporal, en contra de 10 señalado por Nardi (1983).

La utilización de los perfiles cromatográficos de los fenoles en la,discriminación de especies ibéricas y baleáricas del género Asplenium ha sido altamente satisfactoria (Pericás et al., 1985); A. balearicum y A. onopteris son táxones que presentan un perfil cromatográfico distinto. Próximamente se presentarán los resultados cromatográficos obtenidos de los complejos A. adiantum-nigrum y A. obovatum (Rosselló, Salvo, Cubas y Pangua, en preparación).

Aunque A. balearicum puede ser considerado como un endemismo del Mediterráneo Occidental no creemos que dicha corología deba ser considerada como definitiva, ya que probablemente la errónea determinación de pliegos de herbario pertenecientes al complejo A. adiantum-nigrum enmascare la distribución real. De todos modos cabe señalar como curioso el hecho que A. bouharmontii y A. balearicum sean conocidos únicamente hasta el momento en medios insulares: Port Cros, Pantellèria (donde conviven ambos), Palmarola, Cerdeña, Capraia, Ponza y Menorca.

Nardi (1983) supone que esta relativa baja frecuencia distributiva debe relacionarse con una cierta dificultad de expansión a través de sus propios mecanismos reproductores; nosotros creemos que el hecho de la insularidad debe influir, y no en menor medida, en la hasta ahora reducida distribución de la especie ya que, como señalan Mac Arthur \& Wilson (1983), e] aislamiento de ciertas especies en poblaciones adaptadas a hábitats locales y especializados les conferiria a dichas poblaciones una mayor estabilidad y un tiempo de supervivencia mayor, aunque reduciría simultáneamente su capacidad de colonización.

La relativa abundancia de A. balearicum en Menorca puede deberse al hábitat especial que ocupa, que suele ser reducido y 1 a competencia con otros pteridófitos es practicamente nula.

En Menorca no hemos detectado hasta el momento ningún complejo hibridógeno de A. balearicum con sus progenitores o con otros táxones (tal como sucede en Mallorca, por ejemplo, con el A. majoricum Lit.) a pesar de la relativa facilidad en otener dichos híbridos experimentalmente (Shivas, 1969; Lovis et a1., 1972). Creemos que dicho hábitat tan peculiar puede haber servido como una barrera reproductora efectiva, aunque no descartamos que en algunas zonas de ecotono puede hallarse algún retrocruzamiento con A. onopteris.

\section{DESCRIPCION}

Asplenium balearicum Shivas, Brit. Fern Gaz. 10:75, 1969.

Frondes de $(4,5) 5,5-21,6 \mathrm{~cm}$ de longitud y $(1,4) 2,2-8,2 \mathrm{~cm}$ de anchura, de color verde claro. Peciolo de color verde hasta $1 / 3$ de la cara abaxial, el resto castaño, subigual a la lámina; escamas pardas y lanceoladas en la parte inferior de 3,2-5,1 x 0,63-1,05 mm con proyecciones celulares en los márgenes, a veces reflejas; páleas filiformes. Raquis verde en la cara adaxial, verde o castaño en la abaxial, en este último caso no alcanzando la coloración parda $1 / 3$ de la longitud de la lámina. Lámina triangular, raramente deltoide u ovado-lanceo- 


\begin{tabular}{|c|c|c|c|c|}
\hline & $\begin{array}{l}\text { SHIVAS } \\
\text { (1969) }\end{array}$ & $\begin{array}{l}\text { NARDI } \\
(1983)\end{array}$ & $\begin{array}{l}\text { NOGUEIRA \& } \\
\text { ORMONDE } \\
(1986)\end{array}$ & $\begin{array}{l}\text { Presente } \\
\text { trabajo }\end{array}$ \\
\hline $\begin{array}{l}\text { LONGITUD DE } \\
\text { LA FRONDE }(\mathrm{cm})\end{array}$ & 18 & $14-38$ & hasta 18 & $\begin{array}{c}(4,5)-5,5-21,6 \\
(\bar{x}=13,9)\end{array}$ \\
\hline $\begin{array}{l}\text { LONGITUD DE } \\
\text { LA L AMIMA (cm) }\end{array}$ & 11 & $6-16$ & 11 & $\begin{array}{c}(2,5)-3,3-11,7 \\
(\bar{x}=7,5)\end{array}$ \\
\hline $\begin{array}{l}\text { AMCHURA DE } \\
\text { LA LAMIM A (om) }\end{array}$ & 5 & $4-13$ & 6 & $\begin{array}{c}(1,4)-2,2-8,2 \\
(\bar{x}=4,7)\end{array}$ \\
\hline $\begin{array}{l}\text { LONGITUD DEL } \\
\text { PECHOL O(em) }\end{array}$ & 7 & $8-23$ & - & $\begin{array}{c}2-9,7-(11,7) \\
(\bar{x}=6,4)\end{array}$ \\
\hline ESCAMAS (mm) & $\begin{array}{c}4 \times 1 \\
\text { Subulades }\end{array}$ & $\begin{array}{c}\text { hasta } 6 \mathrm{~mm} \\
\text { linear-lanceoladas }\end{array}$ & - & $\begin{array}{c}3,2-5,1 x \\
0,63-1,05 \\
\text { linear-lanceoladas }\end{array}$ \\
\hline $\begin{array}{l}\text { FORMA DE LA } \\
\text { LAMIMA }\end{array}$ & $\begin{array}{l}\text { De estrechamente } \\
\text { triangular a } \\
\text { triangular } \\
\text { Bipinnada }\end{array}$ & $\begin{array}{l}\text { Triangular } \\
\text { (2)-3 pinnada }\end{array}$ & $\begin{array}{l}\text { Estrechamente } \\
\text { triangular } \\
\text { Bipinnada }\end{array}$ & $\begin{array}{l}\text { Triangular, } \\
\text { raramente } \\
\text { ovado-lanceolada } \\
\text { 2-(3) pinnda } \\
\end{array}$ \\
\hline PALEAS & $\begin{array}{l}\text { Similar a las } \\
\text { escamas }\end{array}$ & Filiformes & $\begin{array}{l}\text { Similar a } \\
\text { las escamas }\end{array}$ & $\begin{array}{c}\text { Similar a } \\
\text { las escamas }\end{array}$ \\
\hline RAQUIS & $\begin{array}{c}\text { Castaño } 1 / 2-2 / 3 \\
\text { inferior de la cara } \\
\text { abaxial }\end{array}$ & $\begin{array}{c}\text { Castaño } 1 / 2-2 / 3 \\
\text { inferior de la cara } \\
\text { abaxial }\end{array}$ & $\begin{array}{l}\text { Castaño en la } \\
\text { mitad inferior }\end{array}$ & $\begin{array}{l}\text { Yerde castaño } \\
1 / 3 \text { inferior } \\
\text { de la cara } \\
\text { abaxial }\end{array}$ \\
\hline $\begin{array}{l}\text { PARES DE } \\
\text { PIAWMAS }\end{array}$ & 7 & $5-12$ & 7 & $\begin{array}{c}(4)-6-14-(15) \\
(x=9,4)\end{array}$ \\
\hline $\begin{array}{l}\text { DIMENGSIOWES DE } \\
\text { LAS PIWNAS } \\
\text { MAYORES }\end{array}$ & - & $3-7 \times 2-4$ & - & $\begin{array}{c}0,7-0,39(4,4) \\
\times 0,7-2,4(2,9) \\
(\bar{x}=2,6 \times 1,6)\end{array}$ \\
\hline $\begin{array}{l}\text { PINWUL A PROXIMAL } \\
\text { BASAL DE MAYOR } \\
\text { TAMASO }\end{array}$ & - & Basioscópica & - & Acroscópica \\
\hline $\begin{array}{l}\text { DISPOSICION DE } \\
\text { LOS SOROS }\end{array}$ & $\begin{array}{l}\text { Entre las } \\
\text { nerviaciones y } \\
\text { el margen }\end{array}$ & $\begin{array}{l}\text { Sobre las } \\
\text { nerviaciones }\end{array}$ & $\begin{array}{l}\text { Sobre las } \\
\text { nerviaciones }\end{array}$ & $\begin{array}{l}\text { Sobre las } \\
\text { nerviaciones }\end{array}$ \\
\hline $\begin{array}{l}\text { FORMA DE LOS } \\
\text { SOROS }\end{array}$ & Orados & $\begin{array}{l}\text { Lineares, elipticos } \\
\text { cuando madur os }\end{array}$ & $\begin{array}{l}\text { De orados } \\
\text { a elipticos }\end{array}$ & $\begin{array}{l}\text { Lineares, con el } \\
\text { margen del indusio } \\
\text { débilmente } \\
\text { crenulado }\end{array}$ \\
\hline ESPORAS $(\mu \mathrm{m})$ & $\begin{array}{l}35-45(\bar{x}=42) \\
\text { Perisporio con } \\
\text { papilas esparcidas; } \\
\text { crestas a menudo } \\
\text { anastomosadas }\end{array}$ & $\begin{array}{c}(36)-38-44-(49) \\
(\bar{x}=42)\end{array}$ & $\begin{array}{l}\text { (27) } 35-39(42) \\
\text { Perisporio equinulado } \\
\text { crestado reticulado }\end{array}$ & $\begin{array}{c}(20,5)-22,5-37,5(40) \\
(\bar{x}=32,7) \\
\text { Perisporio papiloso } \\
\text { reticulado, con las } \\
\text { crestas variables y } \\
\text { con microperfo- } \\
\text { raciones }\end{array}$ \\
\hline
\end{tabular}

Tabla 1.- Características morfológicas de A. balearicum según diversos autores. 
lada, de (2,5) 3,3-11,7 cm de longitud, 2(3) pinnada; (4) 6-14 (15) pares de pinnas, subopuestas o a1ternas de ovado-triangulares a triangulares, generalmente más largas que anchas. Pinnas basales más largas que las restantes, aunque algunos ejemplares presentan su máxima anchura a nivel del 2 o par de pinnas; extremidad de las pinnas muy variable, agudas u obtusas, múticas o brevemente caudadas, distintamente dentadas; dientes obtusos, mucronados o cuspidados. Pínnulas ovado-oblongas, 1-2 (3) pares pecioladas; pínnula, proximal acroscópica de cada pinna ligeramente mayor que las otras; pínnulas lobuladas o dentadas. Soros lineares, numerosos, dispuestos sobre las nerviaciones; indusio entero o con el margen crenulado. Esporas monoletas de (20)-22,5-37,5 (40) $\mu \mathrm{m}$ con el perisporio reticulado, con las crestas variables en tamaño y anastomosamiento, papiloso y con microperforaciones dispersas (Figuras 2 y 3 ). Con esporangios de octubre a marzo.

\section{HABITAT}

En Baleares, A. balearicum es conocido unicamente de la zona norte de Menorca, considerándose su presencia como ocasional (Rosselló et al., 1986), si bien en las localidades en que aparece la densidad de sus poblaciones suele ser alta. Prefiere las grietas de los peñascos, en muchos casos próximos al litoral, y en los sitios menos expuestos junto a ejemplares fisuricolas aparecen también otros terricolas, siendo aparentemente indiferente a la orientación. En todos los casos aparece sobre rocas que no presentan carbonatos (areniscas y pizarras paleozoicas y areniscas triásicas) pudiéndose considerar como especie calcifuga. Los suelos sobre los que se desarrolla A. balearicum se aproximan a la textura franca (tabla 2), son moderadamente ácidos y tienen un bajo contenido en sales. E1 porcentaje de materia orgánica puede considerarse como alto; no se han detactado carbonatos y los bicarbonatos oscilan entre 0,3 y 0,7 mequ./1itro.

Vive desde el nivel del mar hasta los 200 metros, conviviendo ocasionalmente con otros pteridófitos tales como Asplenium onopteris L., A. marinum L., Polypodium cambricum L. y Selaginella denticulata (L.) Spring.

\begin{tabular}{|c|c|c|c|c|}
\hline $\begin{array}{l}\text { LOCALIOAD } \\
\text { SITUACION } \\
\text { COMUNIDAD } \\
\text { YEGETAL }\end{array}$ & $\begin{array}{c}1 \\
\text { EE } 7934 \\
\text { Ampelodesmo- } \\
\text { Ericetum scopariae }\end{array}$ & $\begin{array}{c}2 \\
\text { EE } 7934 \\
\text { Ampelodesmo- } \\
\text { Ericetum scodariae }\end{array}$ & $\begin{array}{c}3 \\
\text { FE0362 } \\
\text { Ampelodesmo- } \\
\text { Ericetum scopariae }\end{array}$ & $\begin{array}{c}4 \\
\text { FE } \\
\text { Aro-Phylliretum }\end{array}$ \\
\hline 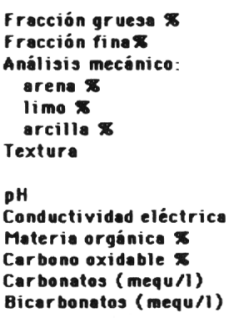 & $\begin{array}{c}12,9 \\
87,1 \\
67 \\
17 \\
16 \\
\text { Franco } \\
\text { arcillo-arenoso } \\
6 \\
269 \\
6,9 \\
3,69 \\
0 \\
0,57\end{array}$ & $\begin{array}{c}4,2 \\
95,8 \\
82 \\
7 \\
11 \\
\text { Franco } \\
\text { arenosa } \\
5,4 \\
205 \\
5 \\
2,87 \\
0 \\
0,30\end{array}$ & $\begin{array}{c}2,9 \\
97,1 \\
\\
81 \\
11 \\
8 \\
\text { Franco } \\
\text { arenoso } \\
6,4 \\
257 \\
3,5 \\
2,01 \\
0 \\
0,43\end{array}$ & $\begin{array}{c}59,2 \\
36,8 \\
\\
50 \\
25 \\
25 \\
\text { Franco } \\
\text { arcilloga } \\
5,8 \\
526 \\
7,1 \\
4,08 \\
0 \\
0,70\end{array}$ \\
\hline
\end{tabular}

Tabla 2.- Caracteristicas edafológicas de tres localidades donde habita A. balearicum: 1,2 - Sa Bassa Verda, 3 - Montgofre Nou, 4 - Favaritx. 


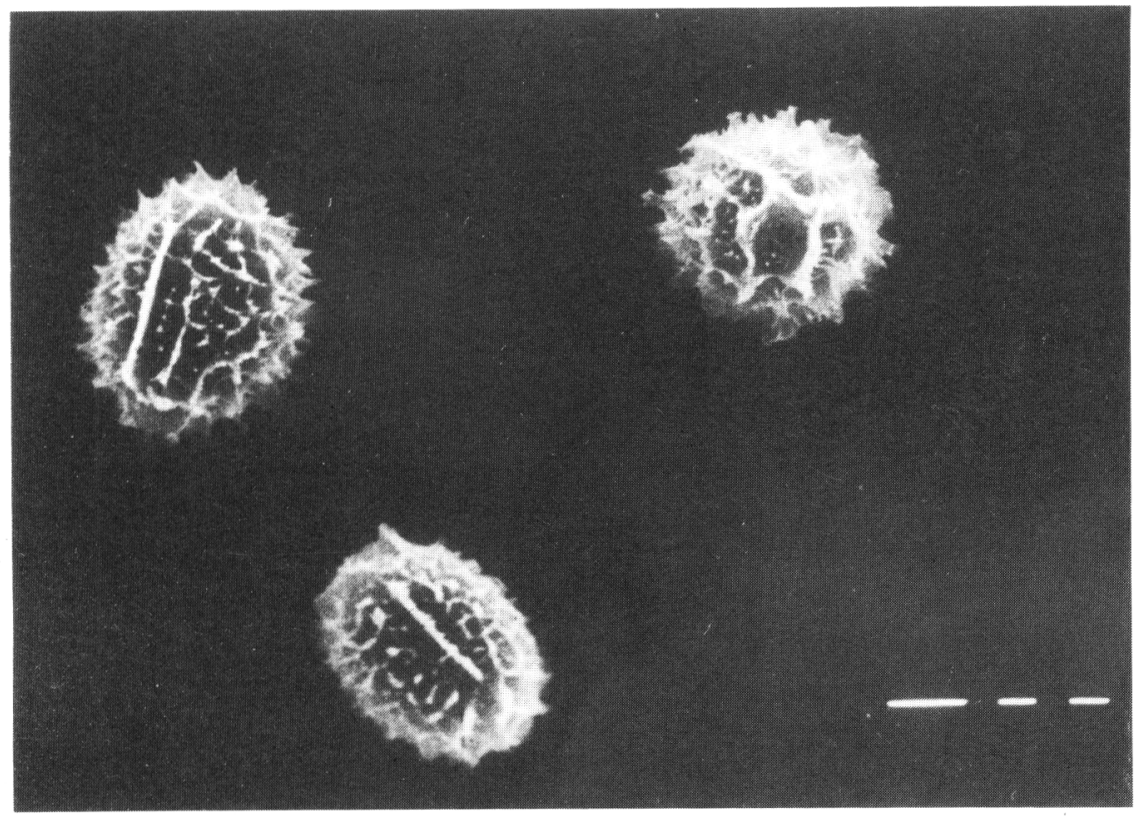

Figura 2.- Esporas de Asplenium balearicum al MEB, procedentes de Favaritx. La barra horizontal de la izquierda corresponde a $10 \mu \mathrm{m}$.

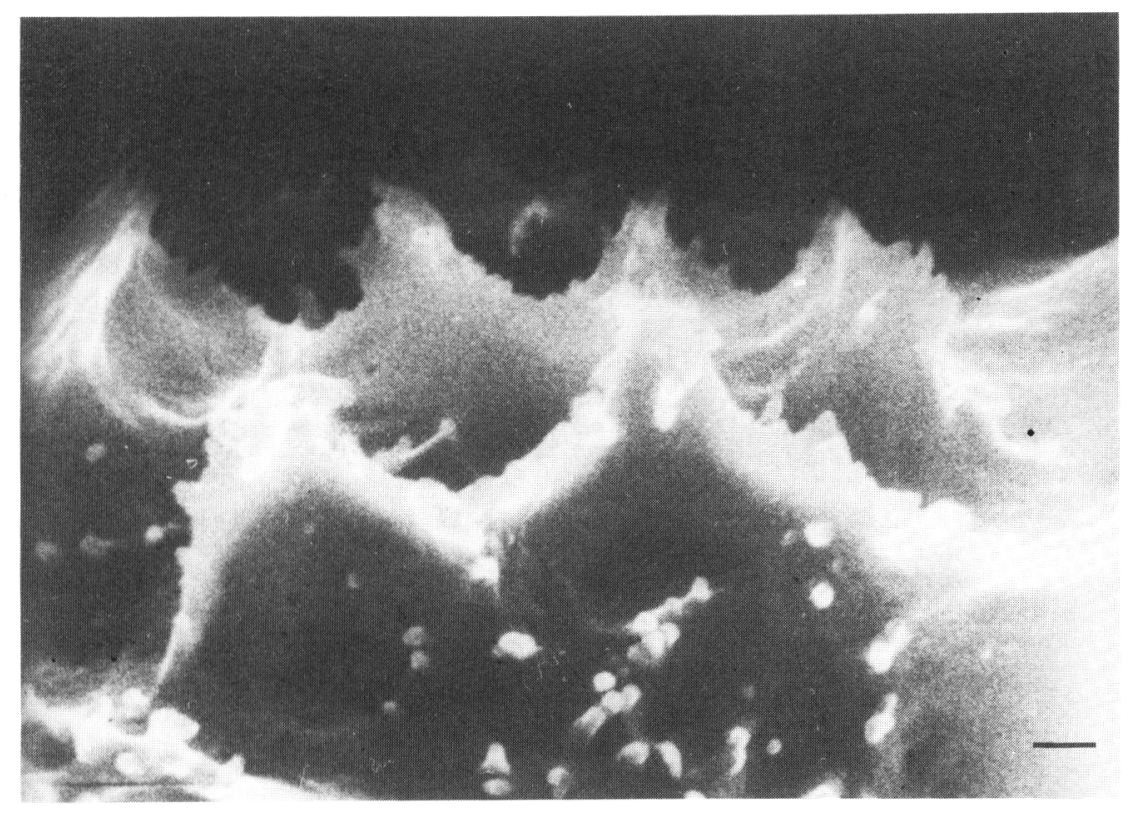

Figura 3.- Detalle del perisporio al MEB, donde se aprecian las papilas y microperforaciones. La barra corresponde a $1 \mu \mathrm{m}$. 
MATERIAL ESTUDIADO

BALEARES: Menorca: Favaritx, grietas de las rocas antes de llegar al faro, 15-XI-84 leg. J.A. Rosselló; Idem XI-85 leg. M. Mus; entre Favaritx y Capifort, grietas y taludes resguardados, 15-XI-84 leg. J.A. Rosselló; Montgofre Nou, grietas de las areniscas, 14-XI-84 leg. J.A. Rosselló; Sa Bassa Verda (Algaierencs), fisuras de las areniscas, XI-85 leg. M. Mus; Sa Vall, en el encinar, XI-85 leg. Mus; Llinaritx Nou, grietas y taludes en el encinar, 13-XI-85 leg. J.A. Rosselló; Binimiel.là, fisuras de los roquedos, 1-II-87 leg. P. Cubas, E. Pangua, J.A. Rosselló; Albufera, Maó, roques sombríes, leg. P. Font Quer, 7-XII-12 (sub A. minoricense Roth.), BC 72070 .

\section{AGRADECIMIENTOS}

Desearíamos expresar nuestra gratitud a P. Cubas, E. Pangua y A.E. Salvo por su apoyo, criticas y ayuda obtenida a lo largo de la realización de este trabajo, así como a M.A. Font, C. Frau, M. Mus y J. Rita por su colaboración.

\section{BIBLIOGRAFIA}

BADRE, F., BOUDRIE, M., PRELLI, R. \& SCHNELLER, J. -1981- Asplenium x sleepiae (A. billotii x A. foresiense) et Asplenium x bouharmontii (A. obovatum $\mathrm{x}$ A. onopteris) Hybrid. nov. (Aspleniaceae, Pteridophyta). Bul1. Mus. Nation. Hist. Nat. (Paris), ser 4, sect. B. 3:473-481.

FERRARINI, E., CIAMPOLINI, F., PICHI SERMOLLI, R.E.G. \& MARCHETTI, D. -1986- Iconographia Palynologica Pteridophytorum Italiae. Webbia $40: 1-202$.

LOVIS, J.D., BROWNSEY, P.J., SLEEP, A. \& SHIVAS, M.G. -1972- The origin of Asplenium balearicum. Brit. Fern Gaz. 10:263-268.

MAC ARTHUR, R.H. \& WILSON, E.O. -1983- Teoría de la Biogeografía insular. Edit. Moll. Monografíes Cientifiques, 2. Palma de Mallorca.

NARDI, E. -1983- Commentaria pteridologica IV. De "Asplenio balearico" Shivas in Italia reperto. Webbia 36:217-223.

NOGUEIRA, I. \& ORMONDE, J. -1986- Asplenium. In Castroviejo et al. (edit): Flora Ibérica. 1:90-104. Madrid.

PERICAS, J., ROSSELLO, J.A. \& SALVO, A.E. -1985- Interés de los estudios cromatográficos en la resolución de problemas taxonómicos y filogenéticos en Pteridófitos Ibéricos. Resúmenes VI Simposio de Botánica Criptogámica: 50. Granada.

ROBERTS, R:H. -1979- Spore size in Asplenium adiantum-nigrum L. and A. onopteris L. Watsonia 12:233-238.

ROSSELLO, J.A., PERICAS, J., ALOMAR, G \& TORRES, N. -1986- Notas peridológicas 6. Atlas Pteridológico de las islas Baleares. Acta Bot. Malacitana 11:294-302.

SHIVAS, M.G. -1969- A cytotaxonomic study of the Asplenium adiantum-nigrum complex. Brit. Fern Gaz. 10:68-80.

Dirección de los autores: J.A. Rosselló, Departamento de Botánica. Facultad de Ciencias. Carretera de Valldemossa km 7,5. 07071 Palma de Mallorca. M. Serra. C/ Muntaner 5, 30. 07003 Palma de Mallorca. 